\section{XGEVA $^{\circledR}$ (Denosumab 120 mg): Überlegene Wirksamkeit in der Prävention von SRE bei soliden Tumoren}

Skelettbezogene Komplikationen (SRE) spielen eine immer wichtigere Rolle in der Osteoonkologie. Die klinischen Symptome zeigen sich in pathologischen Frakturen, starken Schmerzen sowie Rückenmarkskompressionen, die auch bis zur Querschnittslähmung führen können. Oft sind Bestrahlungen, aufwändige Operationen und medikamentöse Therapien nötig. «SRE sind sehr häufig mit mehrwöchigen Krankenhausaufenthalten verbunden», erläuterte PD Dr. Diana Lüftner, Charité Berlin. «Zudem bedeuten sie für die Betroffenen starke Schmerzen bis hin zum Mobilitätsverlust, eine erhebliche Einbuße der Lebensqualität - sowohl für den Patienten selbst als auch für das familiäre Umfeld», betonte Lüftner.

Mit dem voll humanen monoklonalen Antikörper XGEVA $^{\circledR}$ (Denosumab 120 mg) steht seit kurzem ein neuer Therapieansatz mit einem innovativen Wirkmechanismus zur Verfügung.

Denosumab verzögert SRE um zusätzliche 8,2 Monate

Die Marktzulassung von XGEVA beruht auf 3 Head-to-Head Studien der Phase III mit insgesamt mehr als 5700 Patienten, in denen die Wirksamkeit von Denosumab gegenüber Zoledronsäure hinsichtlich der Verzögerung von SRE untersucht wurde. Eine integrierte Analyse aus den 3 Studien belegt die Überlegenheit des neuen Wirkansatzes bei mehr als 50 soliden Tumoren [1]. «Im Vergleich zu Zoledronsäure konnte XGEVA SRE bei Patienten mit fortgeschrittenen soliden Tumoren und Knochenmetastasen signifikant um median 8,2 Monate verzögern», erläuterte Prof. Dr. Ingo Diel, Vorsitzender der Deutschen Osteoonkologischen Gesellschaft (DOG). «SRE traten in den Studien unter XGEVA nicht nur später, sondern insgesamt auch seltener auf: So war das Risiko eines ersten oder nachfolgenden SRE unter
XGEVA um 18\% im Vergleich zu Zoledronsäure verringert», betonte Diel. Die Wirkung von Denosumab zeigte sich in den Studien konsistent über alle SRE-Typen und solide Tumoren hinweg [1].

Schmerzprogression wird verzögert

«Die Behandlung mit Denosumab geht im Vergleich zu Zoledronsäure mit einer verzögerten Schmerzprogression von fast 2 zusätzlichen Monaten einher», erklärte Dr. Friedrich Overkamp, Vorsitzender der Deutschen Gesellschaft für Hämatologie und Onkologie (DGHO). In Folge der besseren Schmerzreduktion mussten bei einer Therapie mit Denosumab weniger Patienten von geringeren Analgetika-Dosen auf starke Opioide umgestellt werden [2]. Der Antikörper wird subkutan alle 4 Wochen in einer Dosis von $120 \mathrm{mg}$ verabreicht.

\section{Günstiges Verträglichkeitsprofil}

Insgesamt betrachtet war die Anzahl unerwünschter sowie schwerwiegender unerwünschter Ereignisse in beiden Behandlungsgruppen vergleichbar. «Akute-Phase-Reaktionen traten unter Denosumab im Vergleich zu Zoledronsäure um $57 \%$ weniger auf», erläuterte Overkamp. Kieferosteonekrosen (ONJ) traten bei 1-2\% der Patienten ohne statistisch signifikanten Unterschied zwischen den beiden Studienarmen auf. Unter XGEVA wurde zwar häufiger eine Hypokalzämie beobachtet, die aber generell nicht symptomatisch oder mit klinischen Folgen verbunden war. Da Denosumab nicht über die Niere ausgeschieden wird, kann auf eine Dosisanpassung bei verminderter Nierenfunktion verzichtet werden.

Positive Studienergebnisse zur Prävention von Knochenmetastasen

In der Phase-III-Studie «147» wurde gezeigt, dass Denosumab das knochenmetastasenfreie

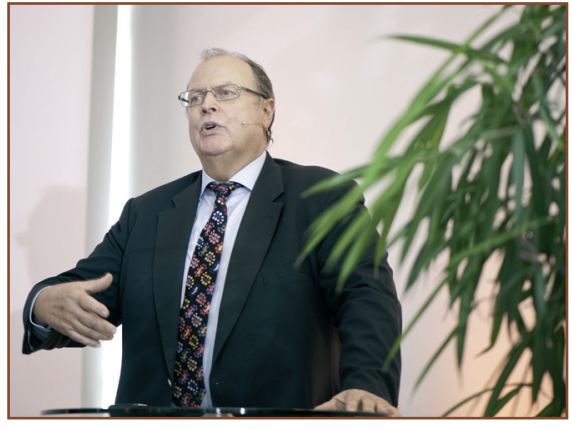

Prof. Dr. Ingo Diel: XGEVA reduzierte das Risiko für SRE im Vergleich zu Zoledronsäure um $18 \%$.

Überleben bei Männern mit kastrationsresistentem Prostatakarzinom, das sich noch nicht auf den Knochen ausgebreitet hat, um 4,2 Monate verlängern konnte [3, 4]. Eine Phase-III-Studie zur adjuvanten Behandlung von Frauen mit frühem Hochrisiko-Mammakarzinom ist in der Rekrutierungsphase. «Wir gehen davon aus, dass dieser Wirkstoff noch einiges an Potenzial bietet», fassten Diel und Overkamp in einem gemeinsamen Ausblick zusammen.

\section{Quelle:}

Launch-Pressekonferenz «XGEVA ${ }^{\circledR}$ - Neue Hoffnung für Patienten mit Knochenmetastasen» der Amgen $\mathrm{GmbH}$, 09.09.2011, München.

\section{Referenzen:}

1 Lipton A et al.: abstr 1249P, ESMO 2010.

2 Cleeland CS et al.: Ann Oncol 2010;21:8s (abstr 1248P).

3 Amgen ${ }^{\circledR}$ Press Release 13. Dezember 2010: www.amgen.com/media

4 Smith MR et al.: Denosumab to prolong bone metastasisfree survival in men with castrate-resistant prostate cancer: results of a global phase 3, randomized, double-blind trial. AUA 2011, Washington, Präsentation.

Weitere Informationen bei

Amgen $\mathrm{GmbH}$

Gerold Krischker

Hanauer Straße 1, 80992 München

gerold.krischker@amgen.com

\title{
PharmaTicker+++ PharmaTicker+++ PharmaTicker+++ PharmaTicker+++
}

Roche. In einer aktuellen Subgruppenanalyse der ATHENA-Studie wurden die Wirksamkeit und das gute Verträglichkeitsprofil von Bevacizumab $\left(\right.$ Avastin ${ }^{\circledR}$ ) speziell für das Kollektiv der älteren und alten Frauen mit fortgeschrittenem Brustkrebs (70 Jahre und älter) bestätigt. Für diese Patientengruppe gab es bislang kaum Daten. Die Subgruppenanalyse dokumentiert, dass es sich bei Bevacizumab um eine gut wirksame und verträgliche Option für die First-line-Behandlung von Frauen mit fortgeschrittenem Brustkrebs in Kombination mit einer meist Taxan-basierten Chemotherapie handelt.

Roche Pharma AG

Dr. H.-U. Jelitto

www.roche.de
ÄZQ / KBV. Das Ärztliche Zentrum für Qualität in der Medizin (ÄZQ) hat im Auftrag der Kassenärztlichen Bundesvereinigung (KBV) 2 neue Kurzinformationen zum Thema Brustkrebs entwickelt: Der Titel «Brustkrebs - was ist das?» richtet sich an Frauen mit Brustkrebs im frühen Stadium, der Titel «Leben mit Brustkrebs» an Betroffene mit fortgeschrittener Erkrankung. Die Informationsblätter stehen niedergelassenen Ärzten zum Ausdrucken und Aushändigen an betroffene Patientinnen auf verschiedenen Plattformen zur Verfügung:

www.patienten-information.de/wartezimmerinformation www.kbv.de/wartezimmerinfo.html www.arztbibliothek.de/wartezimmerinformation
Boehringer Ingelheim gab im August 2011 den Beginn zweier Phase-II-Studien, LUX-Breast 2 und Studie 1200.89, bekannt. Beide Studien prüfen Afatinib bei Patientinnen mit metastasiertem Brustkrebs, bei denen eine Überexpression des erbB2 (HER2) Proteins vorliegt, sogenannte HER2-positive Patientinnen. Klinische Forscher, die an einer Studienteilnahme interessiert sind und dafür möglicherweise geeignete Patientinnen haben, können mit Boehringer Ingelheim Kontakt aufnehmen unter clintriage.rdg@boehringer-ingelheim.com

Boehringer Ingelheim GmbH

Dr. Christina Janista

press@boehringer-ingelheim.de 